Bangladesh J. Bot. 43(1): 37-43, 2014 (June)

\title{
DIFFERENTIAL EXPRESSION PATTERNS OF LIPID GENES IN DEVELOPING SEEDS OF CASTOR BEAN (RICINUS COMMUNIS L.)
}

\author{
Umashankar Chandrasekaran¹ AND Aizhong LiU* \\ Key Laboratory of Tropical Plant Resource and Sustainable Use, Xishuangbanna Tropical \\ Botanical Garden, Chinese Academy of Sciences, 88 Xuefu Road, Kunming,625023, China
}

Key words: Castor bean, Fatty acid, Lipid gene, Expression pattern

\begin{abstract}
Castor bean (Ricinus communis L.) is one of the most important non-edible oilseed crops. Characterization of seed development and lipid accumulation from female flowers pollinated to mature seeds has been carried out. The temporal expression patterns of 65 lipid genes involved in fatty acid and triacylglycerol biosynthesis using the semi-quantitative RT-PCR technique were also investigated. The 65 genes displayed diverse expression patterns which could be collectively grouped into seven groups (I - VII). Four gene groups (I, II, III and V) covering 40 lipid genes presented up-regulated expression patterns, correlating with the rapid lipid accumulation within developing seeds. The present study provides an extended glimpse of the expression patterns of lipid genes involved in fatty acid biosynthesis and triacylglycerol assembly in developing seeds of castor bean, which could serve in variety improvement of castor bean by genetic engineering approach.
\end{abstract}

\section{Introduction}

Castor bean (Ricinus communis L., Euphorbiaceae, $2 \mathrm{n}=20$ ) is an important non-edible oilseed crop and its seed derivatives are often used in aviation oil, lubricants, nylon, dyes, inks, soaps, and adhesive. Among all the vegetable oils, castor bean oil is distinctive due to its high level (over 85\%) of ricinoleic acid (Akpan et al. 2006). Due to an increased demand for castor oil, breeding and improvement of varieties have drawn great attentions from breeders in recent years (Chen et al. 2007). To develop castor as a biodiesel crop, increasing its seed oil yield is one of the major research tasks in practice throughout the world. Enhanced efforts should be paid for the investigating the molecular basis of storage lipid (in the form of triacylglycerol) accumulation during seed development to understand genetic factors regulating storage lipid biosynthesis.

In general, molecular mechanism of storage lipid biosynthesis in higher plants mainly involves two systems: fatty acid (FA) synthesis and triacylglycerol (TAG) assembly. FA biosynthesis initiated by cytosolic acyl-CoA in plastids is sequentially consumed using a substrate, glycerol 3-phosphate (G-3-P) in endoplasmic reticulum (ER), called Kennedy Pathway (Kennedy 1961). In developing seeds, quality and quantity of the storage lipids synthesized depends on the composition of its constituent of TAGs and the number of enzymes which participated in FA biosynthesis and transfer during photosynthesis in leaf and TAG assembly during the Kennedy Pathway (Ohlrogge and Browse 1995). Although a number of lipid genes coding critical enzymes which participated in FA synthesis and TAG assembly were identified in plants, but little is known about the overall expression and regulation profiles of multiple genes involved in lipid biosynthesis from de novo carbon flux to TAG during seed development (Chen et al. 2007). This aspect of storage lipid accumulation is important for attempts to identify the key rate-limiting enzyme genes which regulate storage lipid accumulation during seed development in oil crops. In castor bean, the temporal expression patterns of only a several rate-limiting enzyme genes

*Author for correspondence: <liuaizhong@mail.kib.ac.cn>. ${ }^{1}$ University of Chinese Academy of Sciences, Beijing, China. 
involved in lipid biosynthesis have been studied at different stages of developing seeds (Chen et al. 2007, Cagliari et al. 2010). Hence, in the present study, 65 lipid genes involved in different steps of lipid biosynthesis pathway were identified.

\section{Materials and Methods}

Seeds of castor bean var. ZB306 (provided by Zibo Academy of Agricultural Sciences, Shandong, China) were germinated and grown in the greenhouse of Xishuangbanna tropical botanical garden (Kunming branch) with day temperature at $24-26^{\circ} \mathrm{C}$ and night at $18-20^{\circ} \mathrm{C}$ with the humidity controlled at $60-80 \%$. Hand-pollinated flowers were tagged and recorded as 0 day after pollination (DAP). Capsules were harvested at a 7 day interval from 7 to 63 DAP. Seed samples collected were dissected and frozen immediately in liquid nitrogen and stored at $-80^{\circ} \mathrm{C}$ for further analysis. Seeds dissected at different developing stages were weighed (fresh weight, FW) and were kept for vacuum centrifugation overnight. The dried seed samples were weighed (dry weight, DW). For lipid analysis, total lipid was extracted from seed samples using the hexane-isopropanol method as previously described (Xu et al. 2011). Total RNA was extracted as per the manufacturer's protocol (Takara, Bio.INC, Japan). First-strand cDNA (template) was synthesized using the Prime Script ${ }^{\mathrm{TM}}$ RT-PCR Kit (Takara Bio.INC, Japan) with equal amounts of Oligo dT primer following manufacturer's instructions (Takara Bio.INC, Japan).

Totally, 65 lipid genes (supplementary file) were obtained from the castor bean genome database (http://www.genome.jgi.doe.gov). Based on sequences of the 65 genes, specific primers were designed using Primer Express software version 3.0 (Premier Biosoft INC, CA, USA) with default parameters. RT-PCR amplifications with following PCR conditions were used: initial denaturation at $95^{\circ} \mathrm{C}$ for $1 \mathrm{~min}, 30$ cycles of denaturation at $94^{\circ} \mathrm{C}$ for $30 \mathrm{sec}$, annealing at $55-58^{\circ} \mathrm{C}$ for $20 \mathrm{sec}$ and extension at $72^{\circ} \mathrm{C}$ for $5 \mathrm{~min}$. Castor Ubiquitin gene was used as internal control.

\section{Results and Discussion}

To understand the temporal expression patterns of lipid genes in developing seeds of castor bean, the process of seed development was analysed. The current research showed that the developing seeds grew gradually at the early stage of seed development before ca. 14 DAP. The seed weight (including fresh and dry) increased rapidly after ca. 14 DAP, throughout the middle and late stages of development up to its maximum at ca. 35 DAP. This was followed by a slight decline in seed fresh weight during the late stage of development (after ca. 35 DAP) due to the natural desiccation, whereas the dry weight of seeds slightly increased after ca. 35 DAP (Fig. 1A). Rapid change in seed size occurred before ca. 28 DAP and after ca. 35 DAP a steady decrease in seed size was observed (Fig. 1B). Lipid accumulation trend, observed, was similar to the trend of dry weight changes noticed, i.e. it develops gradually before 21 DAP, and rapidly increases between ca. 21 and 42 DAP, and approaches to its maximum after ca. 42 DAP (Fig. 1A, C). Based on present results, storage lipid accumulation in developing seeds of castor bean mainly occurred in the middle and late stages, in particular between ca. 21 and 42 DAP which correlated with the fresh weight changes during these stages of castor bean seeds (Greenwood and Bewely 1982).

The RT-PCR technique provided a sensitive and specific method to analyze multigenic expressions with minimal concentrations of RNA allowing diverse studies of gene expression (Pray 2008). In relation to this, the present study provide an initial and first glimpse of the patterns of lipid gene expression in developing seeds of castor bean using a semi-quantitative RT-PCR approach, which is critical in understanding the molecular basis of lipid biosynthesis as well as in identifying the key enzyme genes participated in lipid biosynthesis during seed development in castor bean. To determine the expression levels of these genes, the ratio of transcripts of each gene 

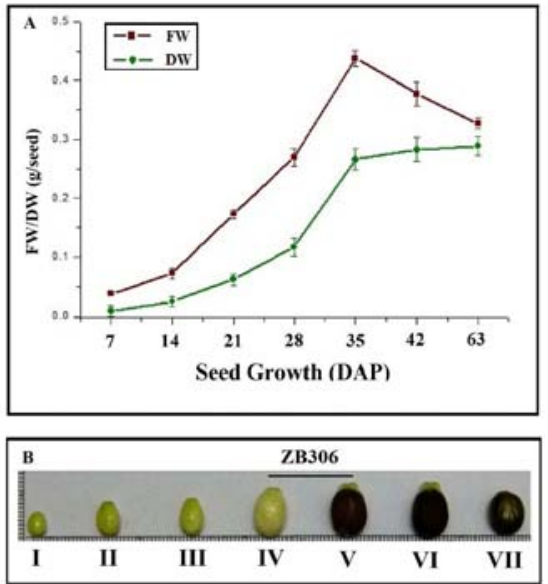

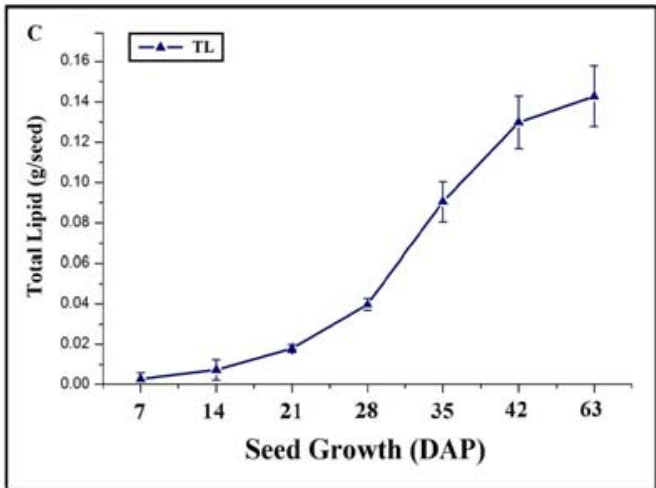

Fig.1. A. Seed weight changes (fresh weight, FW/dry weight, DW) per seed at different stages of development. B. Seed development at different stages after pollination. C. Total lipid changes per seed in developing castor seeds.

to that of castor Ubiquitin gene at different developmental stages was assessed. Based on the relative expression of genes tested, the temporal expression patterns of 65 genes displayed diverse patterns, which could be divided into seven groups (Group I to VII) and (supplementary file). The 21 genes of Group I encode RcBCCP1/RcGPAT6/RcGPAT7/ RcPLA22/ RcPLA23/ RcDGAT2/ RcPDAT1/ RcPDCT/ RcLPAT1/ RcSAD2/ RcFATA/ RcFATB1/ RcFAD6/RcLPCAT/RcACBP/ RcEAR1/RcEAR3/RcHACC/RcKAR/RcPAP3/RcHAD and displayed a concave rise expression pattern from 7 to 63 DAP, with a peak expression at 28,35 or 42 DAP, but low expression at 7 and 63 DAP (Fig. 2A) and (supplementary file). Group II comprised of 8 genes include RcFAD3/RcACP1/RcACP2/RcACP4/RcFATB2/RcKAS2/RcKAS2/RcPDAT3 which showed a linear shaped expression pattern with increase in gene expression particularly from 14 to 42 DAP with a weak or no expression at the early (7 DAP) and late stage (63 DAP) of seed development (Fig. 2B) and (supplementary file).The 6 genes of Group III encode RcKAS1/ RcPLA21/ RcBACC/RcACC1/RcMCAAT/RcBCCP2 and exhibited a high expression at the early stage (7 and 14 DAP) with a linear rise expression pattern in the middle stage (21 to $35 \mathrm{DAP}$ ) and a linear drop expression at the late stage (42 to 63 DAP, with an exception of RcACC1) (Fig. 2C) and (supplementary file). Group IV with 13 genes like RcGPAT2/RcGPAT4/RcPAP1/RcPAP2/ RcACS1/RcACS2/RcACS3/RcACS4/RcPDAT2/RcLPAT3/RcEAR2/RcDGAT1/RcSAD1 displayed high expression only at the early stages (7-21DAP), after which they showed a steady decline throughout seed developmental stages (Fig. 2D) and (supplementary file). Group V with 6 genes included RcACC2/RcSAD3/RcOle1/RcOle2/ RcFAH/RcGPAT1 and showed an expression pattern only during the late stages (28 to 63 DAP) and did not show transcripts expressed (or showed a very low expression) in the early stages (7 to $21 \mathrm{DAP}$ ), (Fig. 2E) and (supplementary file). The 5 genes of Group VI encoded RcSAD4/ RcCPT/ RcPLA2/ RcACP3/ RcGPAT3 and displayed an irregular rise and decline pattern throughout the developmental stages (Fig. 2F) and (supplementary file) with maximal transcript expression observed in the middle to late stages (21 42 DAP). Group VII encoding 6 genes like RcPHA2/RcFATB3/RcPAP4/RcGPAT5/RcLPAT2/ RcLPAT5 were consecutively expressed throughout the seed developmental stages except for the early stage (7 DAP) or the late stage (63 DAP), (Fig. 2G) and (supplementary file). 

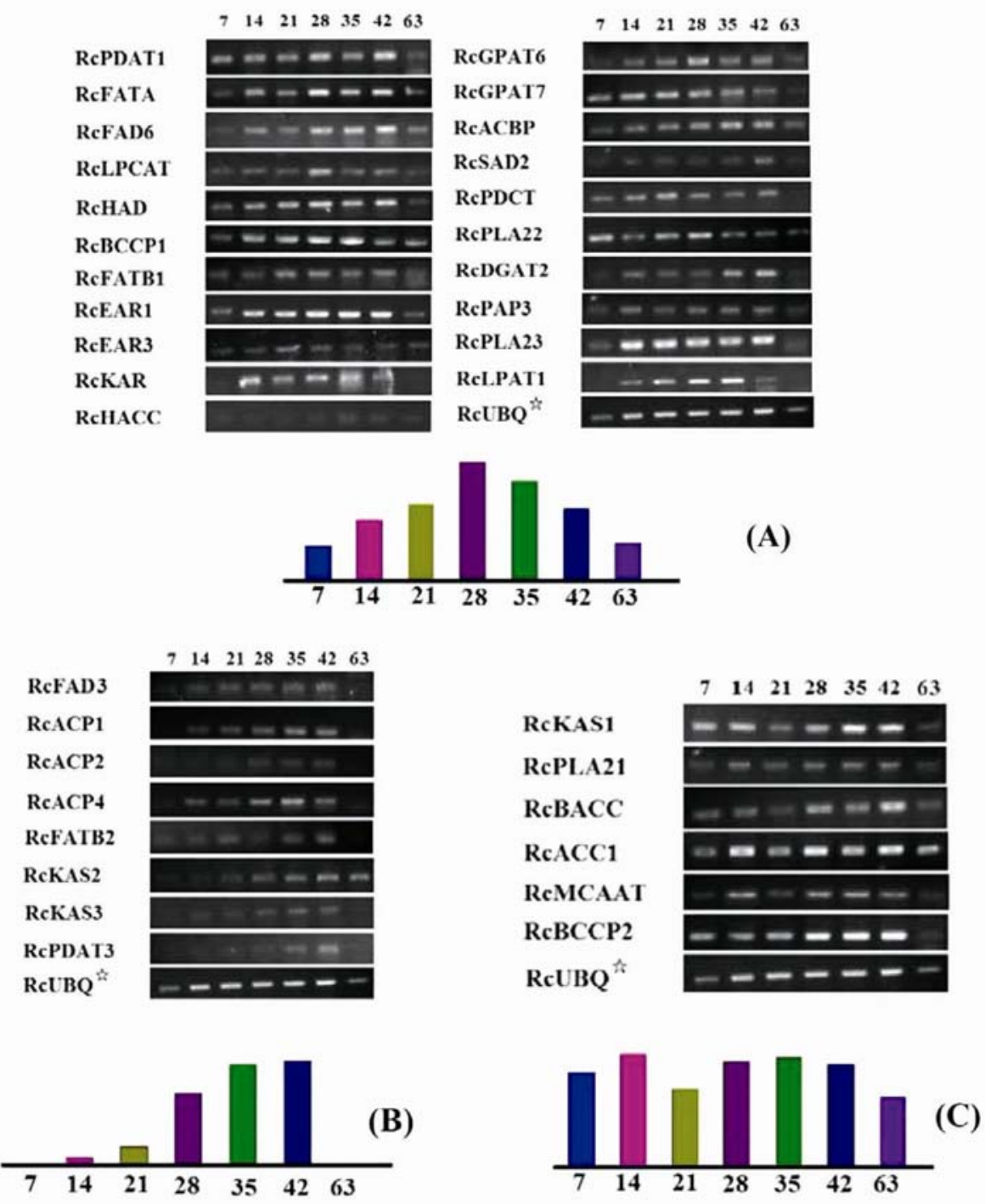

Fig. 2. A, B, C (top). Expression profile of lipid genes (Groups I to III) at different seed developmental stages (DAP). (Bottom) General expression pattern of gene clusters against those of their corresponding control gene: Ubiquitin at different developmental stages.

Investigating the instantaneous expression patterns of genes associated with lipid biosynthesis in the developing seeds contributed in understanding the molecular basis of storage lipid accumulation in plants, though the biosynthesis of storage lipid is complex (Xu et al. 2011). Although the 65 genes tested exhibited various temporal expression patterns within developing seeds, genes clustered in Groups I, II, III and V exhibited similar temporal expression patterns with maximal expression at the middle to late stages of seed development (i.e. between ca. 21 and 42 DAP), when lipids were rapidly accumulated during this period, suggesting the expression of these genes at the transcription level was closely associated with lipid accumulation in developing seeds of castor bean. In particular, RcDGAT2/RcGPAT6/RcGPAT7/RcPDAT1/RcLPAT1/RcPAP3 
and RcLPCAT encoding key enzymes which directly participate in TAG assembly and RcACBP/ RcFATA/RcFATB1/RcBCCP1/RcHACC/RcACC/RcPDCT/RcHAD/RcEAR1/RcEAR2/RcKAR/ RcPLA22 and RcPLA23 encoding key enzymes directly involved in FA biosynthesis by elongating the carbon chain or transferring fatty acids from plastid to ER, were highly expressed indicating that these genes might play a major role in lipid accumulation within developing seeds of castor bean. Besides, present results on the expression pattern of genes like GPAT/ DGAT/ LPAT/ PAP and PDAT was concurrent with that of Chen et al. (2007) and Cagliari et al. (2010) in developing castor seeds. Relatively, previous studies have revealed that genes LPAT (Maisonneuve et al. 2010), DGAT (Andrianov et al. 2009), PDAT (Van Erp et al. 2011), PDCT (Hu et al. 2012), GPAT (Gidda et al. 2003), ACP (Branen et al. 2001), ACBP (Xiao and Chye 2011) and LPCAT (Cagliari et al. 2010) play critical roles in lipid accumulation when engineered in developing seeds of other plants. In support to this, over-expression of the above mentioned lipid (both FA and TAG) genes might directly participate in enhancing overall lipid accumulation in castor seeds. However, further investigation is necessary to characterize the exact involvement of these genes towards lipid biosynthesis in developing castor seeds.

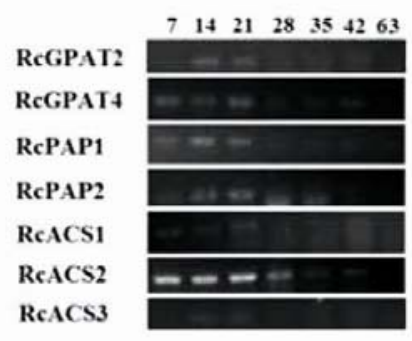

\section{RcACS4 RePDAT2 ReLPAT3 RcE.AR2 RCDGAT RcSAD1 RcUBQ}

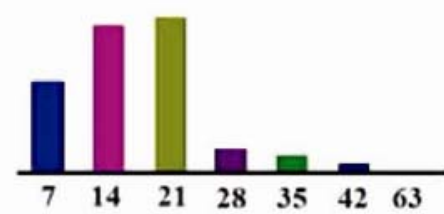

(D)
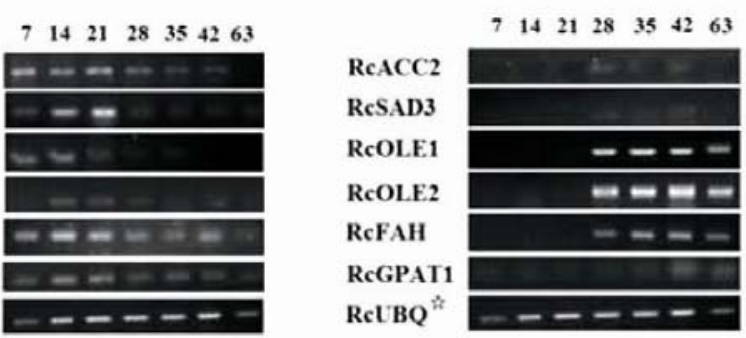
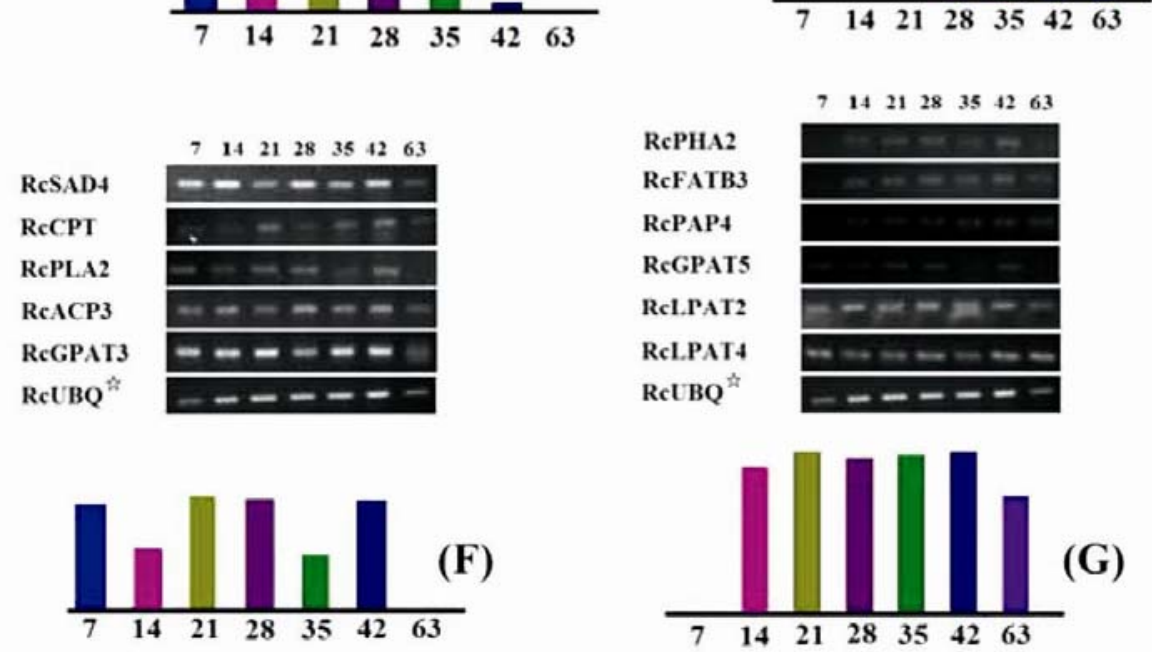

Fig. 3. D, E, F, G (top). Expression profile of lipid genes (Group IV to VII) at different seed developmental stages (DAP). (Bottom) General expression pattern of gene clusters against those of their corresponding control gene: Ubiquitin at different developmental stages. 
In contrary, genes in Group $\mathrm{V}$ were expressed only during middle to late stages of seed development, particularly, the genes RcOle1/ RcOle2 and RcFAH were intensely expressed from 28 to 63 DAP, when fatty acids incorporated into TAG were modified, storage lipids were condensed and oil bodies were rapidly taken shape. Oleosin proteins encoded by oleosin genes were indispensable compositions for the formation of oil body and play a major role in stabilizing the oil bodies and determining the size of oil bodies (Siloto et al. 2006). Over-expression of castor oleosin in Arabidopsis could change the overall fatty acid composition in transgenic Arabidopsis seed modified metabolically to make ricinoleic acid (Lu et al. 2006). Our result on castor oleosin expression was consistent with Chen et al. (2007). During the modification of fatty acids incorporated within TAG, the ricinoleic acids were rapidly accumulated. RcFAH, the only enzyme encoding the hydroxylase responsible for ricinoleate biosynthesis (Lu et al. 2006) was highly expressed at this stage, suggesting the active functioning of RcFAH in this period. Overexpression of castor FAH gene in transgenic Arabidopsis boosted the overall fatty acid composition (Lu et al. 2006) in Arabidopsis seeds. Our study on RcFAH correlated with previous reports (Van De Loo et al. 1995, Chen et al. 2007). Genes clustered in group IV displayed a higher or intense expression at the early stages (before 21 DAP) than at the middle and late stages, implying that these genes might be related to the synthesis of membrane lipids (associated with the rapid increase in size due to cell division) or undergo post-transcriptional regulation mechanism underlying in the synthesis of lipids (Chen et al. 2007). Similar expression pattern was also observed among a group of lipid genes previously studied (Cagliari et al. 2010, Jiang et al. 2012, Chen et al. 2007). Genes in Group VI displayed an irregular rise and decline pattern throughout the developmental stages and genes in Group VII exhibited a constitutive expression pattern throughout the seed developmental stages except for the early stage (7 DAP) or the late stage (63 DAP). Although the genes present in Groups VI and VII, had peak expressions at 28, 35 or 42 DAP, similar pattern to the genes present in Groups I, II, III and V, the extent of their involvement towards TAG accumulation and FA biosynthesis needs to be thoroughly assessed.

Thus, from the present results it is conclusive that most of TAG and fatty acid synthesis genes studied showed consistent expression with the development of storage lipids in castor seeds. Furthermore, the present investigation provides an extended glimpse of the global expression patterns of lipid genes involved in FA biosynthesis and TAG assembly in developing seeds of castor bean, which could facilitate further understanding the molecular mechanisms underlain storage lipid accumulation and serve in improving castor plant by bio-engineering approach.

\section{Supplementary file}

Supplementary file contains the list of primer sequences used in this study (data is available upon request).

\section{References}

Akpan UG, Jimoh A and Mohammed AD 2006. Extraction, characterization and modification of castor seed oil. Leonardo J. Sci. 8: 43-52.

Andrianov V, Borisjuk N, Pogrebnyak N, Brinker A, Dixon J, Spitsin S, Flynn J, Matyszczuk P, Andryszak K, Laurelli M, Golovkin M and Koprowski H 2009. Tobacco as a production platform for biofuel: overexpression of Arabidopsis DGAT and LEC2 genes increases accumulation and shifts the composition of lipids in green biomass. Plant Biotechnology J. 8: 1-11.

Branen JK, Chiou TJ and Engeseth NJ 2001. Over-expression of acyl carrier protein-1 alters fatty acid composition of leaf tissues in Arabidopsis. Plant Physiol. 127: 222-229.

Cagliari A, Margis-Pinheiro M, Loss G, Mastroberti AA, Mariath JEA and Margis R 2010. Identification and expression analysis of castor bean (Ricinus communis L.) genes encoding enzymes from the triacylglycerol biosynthesis pathway. Plant Sci. 179: 499-509. 
Chen GQ, Turner C, He X, Nguyen T, McKeon TA and Laudencia-Chingcuanco D 2007. Expression profiles of genes involved in fatty acid and triacylglycerol synthesis in castor bean (Ricinus communis L). Lipids 42: 263-274.

Gidda KS, Shockey MJ, Rothstein JS, Dyer MJ and Mullen TR 2009. Arabidopsis thaliana GPAT8 and GPAT9 are localized to the ER and possess distinct ER retrieval signals: Functional divergence of the lysine ER retrieval motif in plant cells. Plant Physiol. Biochem. 47:867-879.

Greenwood JS and Bewley JD 1982. Seed development in Ricinus communis L. (castor bean).I descriptive morphology. Can. J. Bot. 60: 1751-1760.

Hu Z, Ren Z and Lu C 2012. The phosphatidylcholine diacylglycerol cholinephosphotransferase is required for efficient hydroxy fatty acid accumulation in transgenic Arabidopsis. Plant Physiol. 158: 1944-1954.

Jiang H, Wu P, Zhang S, Song C, Chen Y, Li M, Jia Y, Fang X, Chen F and Wu G 2012. Global analysis of gene expression profiles in developing physic nut (Jatropha curcas L.) seeds. PLOS one 7: 36522.

Kennedy EP 1961. Biosynthesis of complex lipids. Federation Proc. 20: 934-940.

Lu C, Fulda M, Wallis JG and Browse J 2006. A high-throughput screen for genes from castor that boost hydroxy fatty acid accumulation in seed oils of transgenic Arabidopsis. The Plant J. 45: 845-847.

Maisonneuve S, Bessoule JJ, Lessire R, Delseny M and Roscoe TJ 2010. Expression of rapeseed microsomal lysophosphatidic acid acyltransferase isozymes enhances seed oil content in Arabidopsis. Plant Physiol. 152: $670-684$.

Ohlrogge JB and Browse J 1995. Lipid biosynthesis. Plant Cell 7: 957-970.

Pray L 2008. The biotechnology revolution: PCR and the use of reverse transcriptase to clone expressed genes. Nature Education 1: 1.

Siloto RMP, Findlay K, Lopez-Villalobos A, Yeung EC, Nykiforuk CL and Moloney MM 2006. The accumulation of oleosins determines the size of seed oil bodies in Arabidopsis. Plant Cell 18: 1961-1974.

Van Erp H, Bates PD, Burgal J, Shockey J and Browse J 2011. Castor Phospholipid : Diacylglycerol Acyltransferase facilitates efficient metabolism of hydroxy fatty acids in transgenic Arabidopsis. Plant Physiol. 155: 683-693.

Van De Loo FJ, Broun P, Turner S and Somerville C 1995. An oleate 12-hydroxylase from Ricinus communis L. is a fatty acyl desaturase homolog. Proc. Natl. Ac. Sci. 92: 6743-6747.

Xiao S and Chye ML 2011. New roles for acyl-CoA-binding proteins (ACBPs) in plant development, stress responses and lipid metabolism. Prog. Lipid Res. 50: 141-151.

Xu R, Wang R and Liu A 2011. Expression profiles of genes involved in fatty acid and triacylglycerol synthesis in developing seeds of Jatropha (Jatropha curcas L.). Biomass Bioenerg. 35: 1683-169.

(Manuscript received on 27 November, 2012; revised on 2 October, 2013) 